\title{
Managing the COVID-19 pandemic in non-purpose-built dormitories
}

\section{Dear Editor,}

The rate of coronavirus disease 2019 (COVID-19) infection in the foreign worker (FW) population in Singapore escalated in March 2020. A multiministry task force was set up in April 2020 to address the outbreak in FW residences. ${ }^{1-3} \mathrm{FW}$ residences are highly heterogenous, consisting of purpose-built dormitories and non-purpose-built dormitories (nPBDs). As the medical operations command (MOC) team that planned and executed the medical support plan (MSP) to contain the outbreak and deliver medical care to FWs in nPBDs, we discuss the key elements that served to stem the outbreak.

Unlike purpose-built dormitories that are typically large permanent structures specifically designed to meet FW living needs, nPBDs encompass a wide mix of smaller residences including temporary facilities such as construction temporary quarters and repurposed factory premises (factory-converted dormitories), with occupancies ranging between 10 and several thousand FWs. ${ }^{4}$ The task group for nPBDs managed approximately 300,000 FWs living in approximately 1,300 nPBDs.

The diverse nPBDs and their localities dispersed across Singapore posed a challenge to delivering timely and appropriate care. As the deployment of on-site medical facilities was not feasible, a tiered plan was therefore adopted to efficiently allocate medical resources to nPBDs.

Our MOC team comprised 12 officers from diverse backgrounds - physicians, pharmacists, data analysts and administrators. The MSP comprised 4 key elements: (1) sectoral medical support; (2) mobile medical and nursing teams; (3) telemedicine and vital signs monitoring; and (4) electronic data capture, linkage and analysis. The operations were enabled by provisions in the Infectious Diseases Act and the emergency exceptions of the Personal Data Protection Act. ${ }^{5,6}$

Individual medical teams were deployed at 8 strategically located medical posts to provide sectoral coverage of nPBDs in industrial locations. Medical posts were equiped to deliver primary care and perform COVID-19 swab tests. Mobile medical and nursing teams were deployed to specific dormitories daily, based on a risk stratification algorithm developed by the MOC to identify high risk/needs dormitories. With "circuit breaker" measures-Singapore's version of lockdown - in place, ${ }^{7}$ many FWs with chronic diseases were unable to attend follow-up appointments and obtain prescription refills. As such, nursing teams were deployed to specifically review all FWs above the age of 45 and those with existing chronic diseases, to arrange for their medication.

Telemedicine was made available under a regulatory sandbox by the Ministry of Health Singapore as a 24hour service to all FWs. The simultaneous deployment of a vital signs monitoring application (FWMOM Care mobile app) for all dormitory-dwelling FWs, together with the distribution of pulse oximeters to all nPBDs, allowed twice daily vital signs monitoring (pulse rate and oxygen saturation) and detection of acute respiratory infection symptoms. When preset thresholds were exceeded (e.g. pulse rate above 120 beats per minute or oxygen saturation below 94\%), the application would trigger a teleconsultation.

At the end of each day, data relating to all virtual and in-person consultations - captured via the MW (migrant worker) Health mobile app used by primary care providers to record vital aspects of the patient's condition for infectious disease surveillance-were channelled to the MOC centre. Data analysts processed "report sick" data of FWs, meshing it with telemedicine consultations and data collected by the Forward Assurance and Support Teams (FAST), ${ }^{8}$ along with other secondary datasets on occupancy and age distribution of FWs in nPBDs. FAST comprise officers from the Ministry of Manpower, Singapore Armed Forces and the Singapore Police Force who perform onsite assessments of dormitory facilities. The availability of multifaceted data captured and analysed in near real time allowed for holistic risk assessments of all nPBDs daily, which in turn informed mobile team deployment decisions for the subsequent days. Fig. 1 shows a schematic diagram of the operations undertaken by the MOC team.

At a time when FW movements were restricted, telemedicine had been a useful modality of care delivery to the nPBDs, providing a broad basal level of coverage. ${ }^{9,10}$ Medications were dispatched to stable patients with minor ailments for symptomatic relief. Follow-up telemedicine consultations 3 days after the 


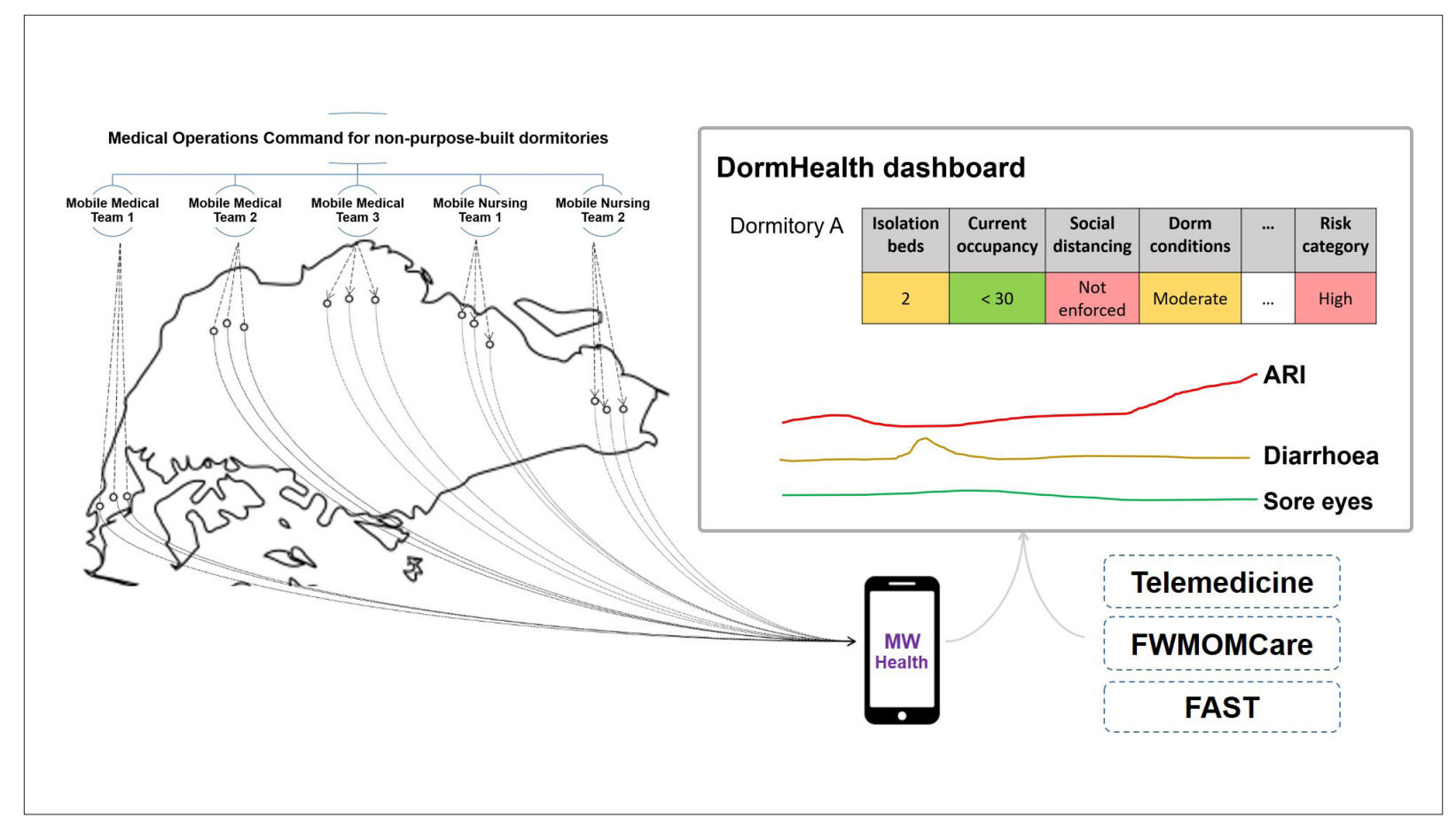

Fig. 1. Overview of operations by medical operations command for non-purpose-built dormitories as well as data capture, transfer and analysis for facilitating dormitory surveillance and decision making. Flow of data from 8 other sectoral medical posts that similarly use the MW Health mobile app to record migrant worker consultations is not shown.

ARI: acute respiratory infection; FAST: Forward Assurance and Support Teams; FWMOMCare: Ministry of Manpower Migrant Worker Care mobile app; MW Health: Migrant Worker Health mobile app.

initial consultation allowed patients to be discharged if symptoms were resolved.

While telemedicine played a key role, a limited physical examination meant that FWs who needed more thorough assessments had to be sent to the sectoral medical posts in a timely manner. ${ }^{11}$ These posts (with some having in situ isolation facilities) provided the next layer of care, particularly for patients with acute respiratory infection symptoms. However, because of movement controls, dormitory operators were only allowed to send FWs to medical posts via a dedicated transport service. Timely communication of the medical needs of a worker following a telemedicine consult therefore became an important function of the MOC, to ensure efforts were coordinated and FWs received timely care. Providers could also activate ambulance services for FWs who were deemed to require urgent tertiary care.

The MOC mobile team deployments were primarily aimed at high-risk/needs dormitories. Nursing teams were deployed in dormitories where needs were less acute, while medical teams were deployed in dormitories with high numbers of COVID-19 cases. In scenarios where FWs were unable to leave their dormitories (e.g. varicella-zoster virus infection), a mobile medical team was deployed instead to review the FW on-site. Although the majority of FWs living in dormitories were relatively young and were of a certain level of physical fitness, the combination of the tiered, care delivery plan along with testing, conveyance and isolation strategy likely contributed to the very low numbers of COVID-19-infected FWs needing tertiary care.

The adopted MSP has been able to address the needs of FWs housed in 1,300 geographically dispersed nPBDs. Telemedicine and electronic data collection and analysis have been pivotal technology enablers in meting out the MSP. Coordination and communication between the MOC and Ministry of Manpower's operations team have also played a key role in addressing the needs of FWs in nPBDs. Having identified the requirements for servicing nPBDs; the data flow from both Ministry of Health and Ministry of Manpower; and the linkages for surveillance, we are now better placed to define resource requirements to address the current pandemic and prevent future COVID-19 outbreaks. 


\section{REFERENCES}

1. Woo JJ. Policy capacity and Singapore's response to the COVID-19 pandemic. Policy and Society 2020;39:345-62.

2. Young BE, Ong SWX, Kalimuddin S, et al. Epidemiologic features and clinical course of patients infected with SARS-CoV-2 in Singapore [published correction appears in JAMA 2020;323:1510]. JAMA 2020;323:1488-94.

3. Ministry of Manpower. Measures to contain the COVID-19 outbreak in migrant worker dormitories, 14 December 2020. Available at: https://www.mom.gov.sg/newsroom/pressreleases/2020/1214-measures-to-contain-the-covid-19-outbreak-inmigrant-worker-dormitories. Accessed on 20 December 2020.

4. Ministry of Manpower. Various types of housing and their specific requirements. Available at: https://www.mom.gov.sg/passes-andpermits/work-permit-for-foreign-worker/housing/various-types-ofhousing. Accessed on 1 July 2020

5. Singapore Statutes Online. Infectious Diseases Act (Chapter 137), 6 August 2021. Available at: https://sso.agc.gov.sg/Act/IDA1976. Accessed on 6 August 2021.

6. Singapore Statutes Online. Personal Data Protection Act 2012, 6 August 2021. Available at: https://sso.agc.gov.sg/Act/PDPA2012. Accessed 6 August 2021.

7. Ministry of Health Singapore. Circuit breaker to minimise further spread of Covid-19. https:/www.moh.gov.sg/news-highlights/details/ circuit-breaker-to-minimise-further-spreadof-covid-19. Accessed on 1 July 2020.

8. Government of Singapore. Pulling together FAST to ensure the well-being of migrant workers. 19 May 2020.; https://www.gov.sg/ article/pulling-together-fast-to-ensure-the-well-being-of-migrantworkers. Accessed on 5 August 2021.

9. Hollander JE, Carr BG. Virtually perfect? Telemedicine for Covid-19. N Engl J Med 2020;382:1679-81.

10. Ting DSW, Carin L, Dzau V, et al. Digital technology and COVID-19. Nat Med 2020;26:459-61.

11. Monaghesh E, Hajizadeh A. The role of telehealth during COVID-19 outbreak: a systematic review based on current evidence. BMC Public Health 2020;20:1193.
Si Jack $\underline{\text { Chong }}{ }^{1}{ }_{F A M S}$, Sreemanee Raaj Dorajoo ${ }^{2}$ PhD, Seng Bin Ang ${ }^{3}$ MMed, Iain Beehuat Tan ${ }^{4,5}{ }_{\text {MRCP }}$, Clive Tan ${ }^{6,7}{ }_{M P H}$, Kok Pun Foong ${ }^{6} \mathrm{MSc}$, Jui Sheng Choo $^{8}{ }_{M B A}$, Li Yang Hsu ${ }^{7}{ }_{M P H}$, Weilong Yeo ${ }^{2}{ }_{B S c}{ }_{\text {(Hons }) \text {, Eti Bhasker }}^{9}{ }^{9}$ PP, Chun Shan Goh ${ }^{10}{ }_{B S C}$ (Hons), Saihah Ismadi ${ }^{11}{ }_{B B A}$, Cherng Yeu Neo ${ }^{12}$ BSC (Hons),

Michael Tack Keong Wong ${ }^{13}$ MMed

\footnotetext{
${ }^{1}$ Department of Plastic Reconstructive and Aesthetic Surgery, Singapore General Hospital, Singapore

${ }^{2}$ Vigilance \& Compliance Branch, Health Products Regulation Group,

Health Sciences Authority, Singapore

${ }^{3}$ Family Medicine Service, KK Women's and Children's Hospital, Singapore

${ }^{4}$ Department of Medical Oncology, National Cancer Centre Singapore, Singapore

${ }^{5}$ Genome Institute of Singapore, Agency for Science, Technology and Research, Singapore

${ }^{6}$ Goh Keng Swee Command and Staff College, Singapore Armed Forces, Singapore

${ }^{7}$ Saw Swee Hock School of Public Health, National University of Singapore, Singapore

${ }^{8}$ Primary and Community Care Development Division, Agency for Integrated Care, Singapore

${ }^{9}$ Data Governance Division, Ministry of Health, Singapore

${ }^{10}$ Assurance, Care and Engagement, Ministry of Manpower, Singapore

${ }^{11}$ Workplace Safety and Health Institute, Ministry of Manpower, Singapore

${ }^{12}$ Centre for Regulatory Excellence, Duke-NUS Medical School,

Singapore

${ }^{13}$ SingHealth Regional Health System, SingHealth, Singapore
}

Correspondence: Dr Sreemanee Raaj Dorajoo, Vigilance \& Compliance Branch, Health Products Regulation Group, Health Sciences Authority, 11 Biopolis Way, \#11-01 Helios, Singapore 138667.

Email: sreemanee dorajoo@hsa.gov.sg 